\title{
A Novel Method of Implant Coverage for Post-Mastectomy Reconstruction after Previous Augmentation: A Case Report
}

\author{
Elisabetta Maria Cristina Rossi ${ }^{a} \quad$ Alessandra Invento $^{a}$ Marco lera $^{c}$ \\ Virgilio Sacchini ${ }^{a, b}$ Paolo Veronesi ${ }^{a, b}$ Francesca De Lorenzi ${ }^{c}$

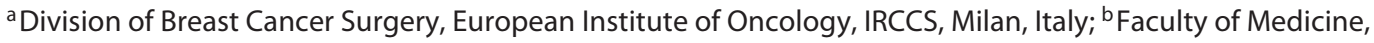 \\ University of Milan, Milan, Italy; ' Division of Breast Cancer Reconstruction, European Institute of Oncology, IRCCS, \\ Milan, Italy
}

\section{Established Facts}

- With the increasing use of breast augmentation, the number of breast cancer patients with a prior history of augmentation is also likely to increase.

- Evolving patterns in mastectomy technique and immediate implant-based reconstruction highlight the need for outcomes assessing the clinical implications of prior implant placement.

\section{Novel Insights}

- A novel capsular flap of the pre-existing old capsule is described as a reliable source of tissues for implant coverage after post-mastectomy reconstruction in augmented patients.

\section{Keywords}

Breast cancer $\cdot$ Augmentation mammaplasty $\cdot$ BRCA patient

\section{Abstract}

Background: Breast augmentation is the most common cosmetic surgical procedure in the USA, with nearly 300,000 women undergoing surgery annually. National incidence rates predict that among women undergoing breast augmentation each year, approximately 35,000 will eventually be diagnosed with breast cancer, in particular individual $B R C A 1 / 2$ germline mutant carriers. Case Report: Our case introduces a novel method of implant coverage after immediate post-mastectomy reconstruction in augmented patients. A novel "capsular flap" (flap of the pre-existing old capsule) is isolated and refolded to cover the outer lower portion of the implant. Conclusion: Tailored surgical approaches can be offered to those patients previously augmented and requiring mastectomy after breast cancer diagnosis.

๑) 2020 S. Karger AG, Basel

\section{Introduction}

Individual $B R C A$-mutated carriers have an increased risk of breast and ovarian cancer at a relatively young age [1]. BRCA-mutated carriers and women with a family history of breast carcinoma have an estimated lifetime risk of $45-67 \%$ [2, 3]. Bilateral preventive mastectomy is recommended to reduce this risk. 
Fig. 1. Schematic drawing of the "turnover" capsular flap. a The old posterior capsule adherent to the pectoralis major muscle. b Flap harvested from the pectoralis major muscle. c Complete coverage of the outer inferior part of the implant.
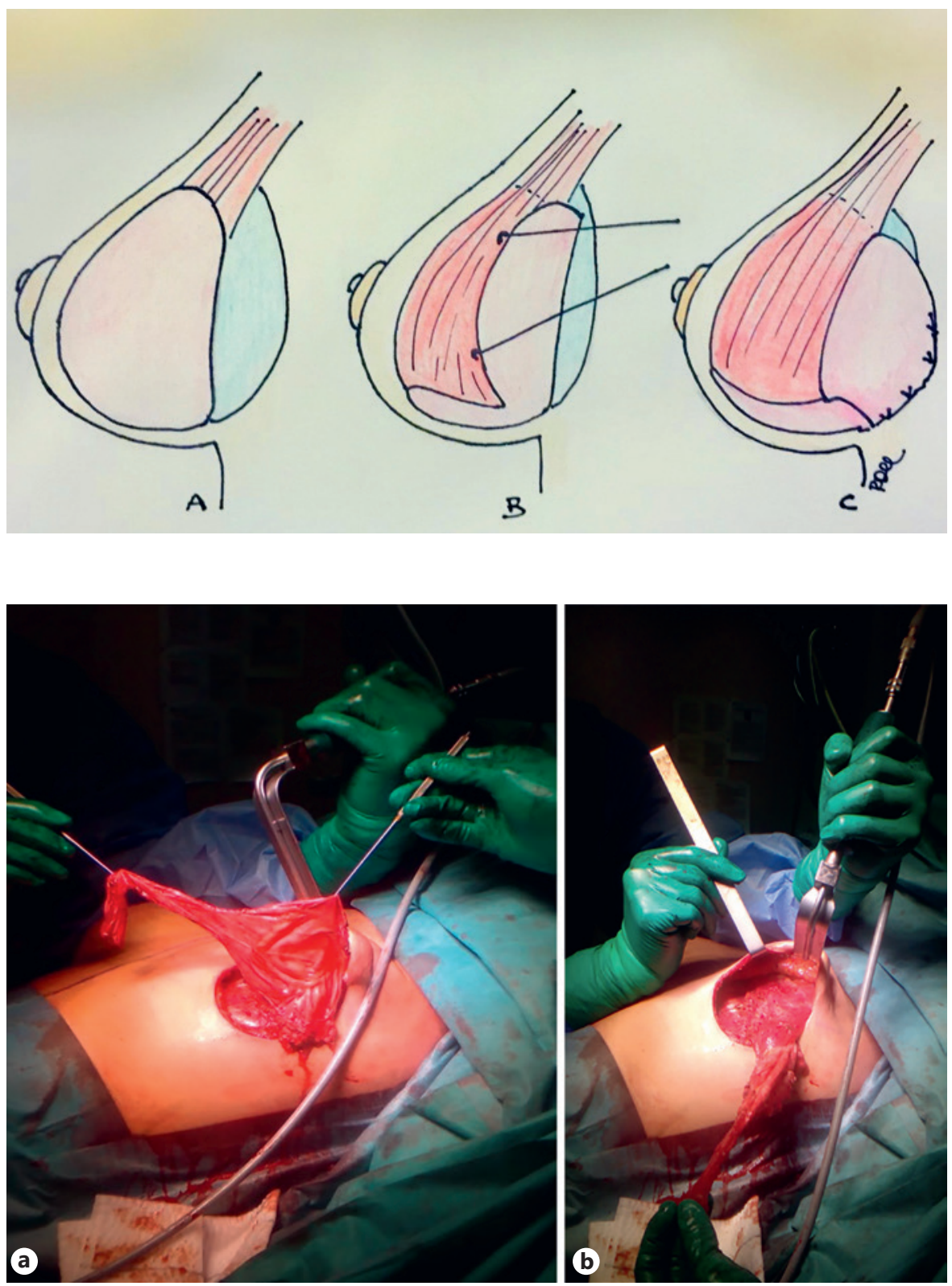

Fig. 2. a, b Relaxation of the fibrous capsule, creating a capsular "flap."

With the increasing number of breast augmentation, the number of breast cancer patients with a prior history of augmentation is also likely to increase.

McCarthy et al. [4] showed improvement in the quality of life with bilateral preventive mastectomy and immediate reconstruction.

Our paper shows a novel technique of implant coverage in post-mastectomy implant-based reconstruction after augmentation mammoplasty in a BRCA1 mutation carrier.

\section{Clinical Case}

A 30-year-old woman with bilateral subglandular prostheses (round shaped, $490 \mathrm{~mL}$ ) presenting a right breast tumor was admitted to our department. Preoperative biopsy revealed an invasive ductal breast carcinoma, ER 80\%, PgR 50\%, HER2 ++, Ki 67
$30 \%$. Considering age and her positive family history, she underwent genetic counseling and genetic test: she resulted BRCA1 germline mutant carrier, with pathogenic role. She was candidate for right nipple-sparing mastectomy, sentinel lymph node biopsy, and left preventive nipple-sparing mastectomy. A two-team approach was used, including a breast surgeon and a plastic surgeon. Mastectomies were performed through a skin incision at the inframammary fold. The right sentinel lymph node was metastatic, and complete axillary dissection was performed. The right nipple-areola complex was spared because no cancer cells were found behind the nipple on frozen section. Previous subglandular prostheses were bilaterally removed at the time of mastectomy en bloc with the anterior surface of their "old capsules," which was left adherent to the breast parenchyma. The posterior surface of each old capsule was preserved intact and adherent to the pectoralis major muscle on both sides. A subpectoral pocket was harvested without harvesting the serratus anterior muscle.

Afterwards, the posterior surface of the old capsules was carefully separated from the pectoralis major muscle from the medial 
Fig. 3. a, b Immediate postoperative outcome.
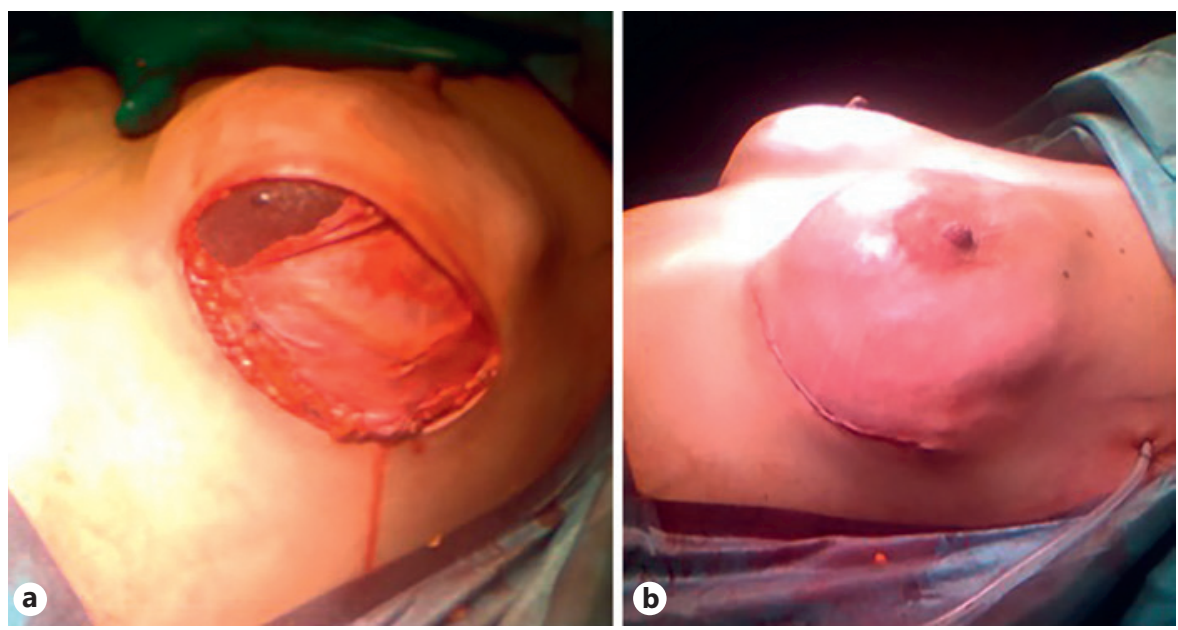

side towards the lateral part. A strip of the old posterior capsule was maintained, fused at the lateral part of the pectoralis major muscle for about $2 \mathrm{~cm}$ in width. The capsule was then refolded and rotated laterally in a "turnover" fashion, creating a capsular flap and allowing the coverage of the outer lower portion of the new implant (Fig. 1). Allergan style 410 MX $550 \mathrm{~g}$ implants were used bilaterally. The old capsule was sutured laterally to the medial border of the serratus anterior muscle, achieving the lateral control and complete closure of the pocket (Fig. 2, 3). Two drains were placed in the right breast, and one drain on the left side. Subdermal and skin layers were sutured with Monocryl 3/0 and 4/0.

Final tumor staging was invasive ductal carcinoma, $\mathrm{pT} 1 \mathrm{~b} \mathrm{pN} 1 \mathrm{a}$ (1/25) G3. At multidisciplinary postsurgical meeting, adjuvant chemotherapy and hormone therapy were recommended for the patient. No postoperative complications occurred (Fig. 4).

\section{Discussion}

We present a case report of breast cancer occurring after bilateral subglandular augmentation. Nipple-areola-sparing mastectomies were performed with a skin incision at the level of the inframammary fold. We used the previous "augmentative" scars hidden in the fold, not creating further scarring.

In case of augmentation with submuscular implants, at the time of mastectomy, the old pocket is easily enlarged by multiple capsulotomies. In case of subglandular augmentative implants, the change of the surgical plane (from subcutaneous to submuscular) may be necessary even in the era of prepectoral post-mastectomy reconstructions.

Although the use of the old "augmentative" implant capsule at the time of mastectomy has already been described as an extension of the pectoralis major muscle for inferior pole coverage [5], to our knowledge, it has never been described in the "turnover" fashion in case of previous subglandular implants. In these specific cases, it has been demonstrated that preserving the posterior surface of the old

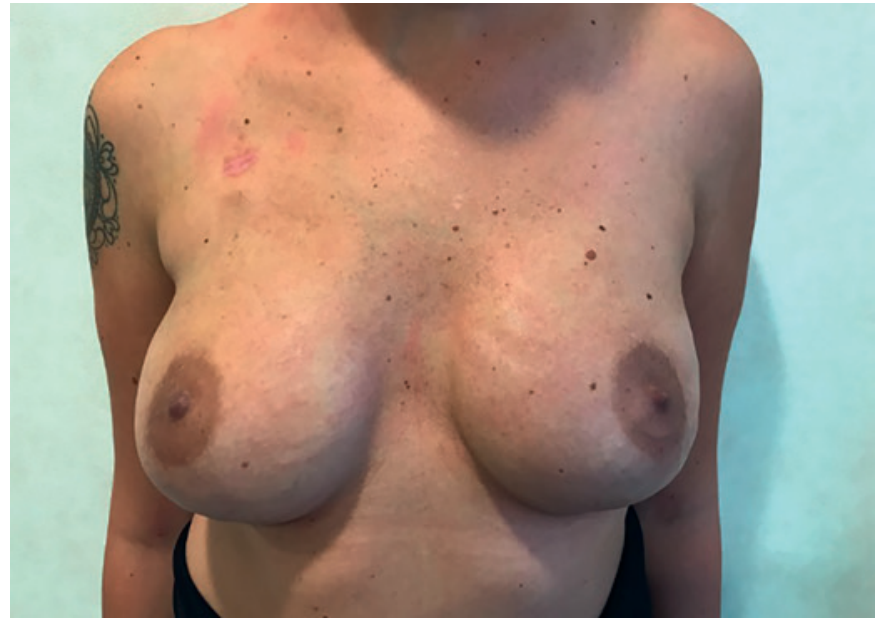

Fig. 4. Postoperative result at 6 months.

capsule untouched facilitates the dissection of the new muscular pocket behind the pectoralis major muscle [6].

According to our experience, capsular flaps (flaps of the pre-exiting implant capsule) can be considered a reliable source of tissue not only to facilitate the creation of the subpectoral pocket but they may also enlarge the pocket itself. In fact, in our particular case, the capsular flap was used as a dermal matrix or synthetic mesh to cover the outer inferior part of the implant.

In our experience, the main advantages of this technique are the following:

- The capsular flap allows the immediate placement of the suitable definitive prosthesis, allowing the maintenance of breast volume similar to the preoperative situation, as desired by the majority of patients who have decided for breast augmentation. The conventional submuscular fascial pocket would probably not be large enough for the placement of such a large implant or to achieve complete lateral coverage of the implant. 
- As biological matrices or synthetic meshes, the capsular flap is interposed between the implant and the mastectomy flap, therefore supporting and stabilizing the implant and reducing the incidence of implant exposure.

- It avoids the use of matrices and meshes, and therefore the complications related to foreign materials.

- Compared to matrices and meshes, our technique is cost-effective, since the patient is of course not charged with any cost for the native old capsule.

On the contrary, this technique also has limitations. In case of a thinner native capsule, pre-existing capsular flap dissection could be very difficult or technically impossible to harvest. We recommend at least about $2-3 \mathrm{~mm}$ capsule thickness, which can be managed more easily. Therefore, this "turnover capsular flap" is not indicated in all previously augmented patients having their implants in the subglandular space.

Any plastic surgeon familiar with breast surgery can easily approach this technique, the most difficult step being the careful harvesting of the capsular flap from the pectoralis major muscle. The learning curve consists of approximately 1-2 cases, and it can easily be taught to any surgeon familiar with breast surgery, even to residents and fellows. No specific surgical skills are required.

Finally, Zingaretti et al. [7] reported the use of meshed autologous dermal matrix to cover the inferior pole of large implants after mastectomy. The autologous dermis is harvested from the contralateral breast in patients having simultaneous breast reduction/mastopexy. Meshing the autograft ensures the increase of the graft surface area to provide additional draining and to improve the engraftment of the autologous dermal matrix.

Our technique could be an alternative for augmented patients surely not requiring any breast reduction. Meshing the pre-existing capsule is not possible (it is partially left adherent to the pectoralis major muscle) and even unnecessary. In fact, the capsular flap is long enough to cover the outer inferior part of the implant in every case, since it relies on the old capsule at the inferior pole. Additional drainage is not necessary if two drains are placed, one draining the mastectomy space and the axilla and the other one draining the muscular-capsular pocket.

\section{Conclusion}

Capsular flaps (flaps of the pre-existing implant capsule) are definitively a reliable source of tissue to facilitate post-mastectomy reconstructions in augmented patients. We present a new "turnover" capsular flap to cover the outer inferior portion of the implant in those patients who previously underwent subglandular augmentation.

Future studies are needed to compare matrix and capsular flap performances in the long term to provide the best surgical approach to augmented women requiring mastectomy, including $B R C A$ mutant carriers.

\section{Statement of Ethics}

The procedures performed in this study involving human participants were in accordance with the ethical standards of the institutional and national research committee and with the 1964 Helsinki Declaration and its later amendments or comparable ethical standards.

The patient gave her written informed consent to publish her case.

\section{Disclosure Statement}

The authors have no conflicts of interest to declare.

\section{References}

1 Harmsen MG, Hermens RP, Prins JB, Hoogerbrugge N, de Hullu JA. How medical choices influence quality of life of women carrying a BRCA mutation. Crit Rev Oncol Hematol. 2015 Dec;96(3):555-68.

2 Giuliano AE, Boolbol S, Degnim A, Kuerer H, Leitch AM, Morrow M. Society of Surgical Oncology: position statement on prophylactic mastectomy. Approved by the Society of Surgical Oncology Executive Council, March 2007. Ann Surg Oncol. 2007 Sep;14(9):2425-7.

3 Antoniou A, Pharoah PD, Narod S, Risch HA, Eyfjord JE, Hopper JL, et al. Average risks of breast and ovarian cancer associated with
BRCA1 or BRCA2 mutations detected in case Series unselected for family history: a combined analysis of 22 studies. Am J Hum Genet. 2003 May; 72(5):1117-30.

4 McCarthy CM, Hamill JB, Kim HM, Qi J, Wilkins E, Pusic AL. Impact of Bilateral Prophylactic Mastectomy and Immediate Reconstruction on Health-Related Quality of Life in Women at High Risk for Breast Carcinoma: Results of the Mastectomy Reconstruction Outcomes Consortium Study. Ann Surg Oncol. 2017 Sep;24(9):2502-8.

5 Veronesi P, De Lorenzi F, Loschi P, Rietjens $\mathrm{M}$, Veronesi U. Current Trends in the Onco- logic and Surgical Managements of Breast Cancer in Women with Implants: Incidence, Diagnosis, and Treatment. Aesthetic Plast Surg. 2016 Apr;40(2):256-65.

6 Salgarello M, Rochira D, Barone-Adesi L, Farallo E. Immediate breast reconstruction after skin- or nipple-sparing mastectomy for previously augmented patients: a personal technique. Aesthetic Plast Surg. 2012 Apr;36(2): 313-22.

7 Zingaretti N, Guarneri GF, De Biasio F, Shoeib MA, Parodi PC. The Use of Meshed Dermal Autograft in Breast Reconstruction. Aesthetic Plast Surg. 2018 Dec;42(6):1704-6. 